\title{
Temporal parallelization of edge plasma simulations using the parareal algorithm and the SOLPS code
}

\author{
D. Samaddar ${ }^{\mathrm{a}, *}$, D.P. Coster ${ }^{\mathrm{b}}$, X. Bonnin ${ }^{\mathrm{c}, \mathrm{d}}$, C. Bergmeister ${ }^{\mathrm{a}}$, E. Havlíčkováa ${ }^{\text {, }}$ \\ L.A. Berry ${ }^{\mathrm{e}}$,W.R. Elwasif ${ }^{\mathrm{e}}$, D.B. Batchelor ${ }^{\mathrm{e}}$ \\ ${ }^{a}$ CCFE, Culham Science Centre,Abingdon, Oxon, OX14 3DB, UK \\ ${ }^{b}$ Max-Planck-Institut für Plasmaphysik, Germany \\ ${ }^{c}$ CNRS-LSPM, Université Paris 13, Sorbonne Paris Cit, Villetaneuse,France \\ ${ }^{d}$ ITER Organization, Route de Vinon sur Verdon, 13115 St Paul Lez Durance, France \\ ${ }^{e}$ Oak Ridge National Laboratory, Oak Ridge, USA
}

\begin{abstract}
It is shown that numerical modelling of edge plasma physics may be successfully parallelized in time. The parareal algorithm has been employed for this purpose and the SOLPS code package coupling the B2.5 finite-volume fluid plasma solver with the kinetic Monte-Carlo neutral code Eirene has been used as a test bed. The complex dynamics of the plasma and neutrals in the scrape-off layer (SOL) region makes this a unique application. It is demonstrated that a significant computational gain (more than an order of magnitude) may be obtained with this technique. The use of the IPS framework for event-based parareal implementation optimizes resource utilization and has been shown to significantly contribute to the computational gain.

Keywords: time parallelization, parareal algorithm, edge plasma, scrape off layer (SOL), SOLPS, Eirene, magnetically confined plasmas
\end{abstract}

PACS: 02.70.-c, 52.40.Hf, 52.55.Fa, 52.65.-y, 02.90.+p, 07.05.Tp

\section{Introduction}

One of the biggest challenges in computational science is often to find the optimum balance between feasible wallclock time and the complexity of the sim-

\footnotetext{
* Corresponding author

Email address: Debasmita.Samaddar@ukaea.uk (D. Samaddar)
} 
ulation. Modern supercomputers with ever increasing number of cores require codes that sufficiently scale to maximize resource utilization. Parallelizing these computations is an option - and traditional techniques of parallelization, such as space parallelization indeed reduce the computational time, but leave a lot of room for further improvement. This is often because the computational gain reaches saturation and increasing the number of processors does not necessarily improve the gain beyond a certain point. There are also parts of the codepackages where standard parallelization does not have a large impact and hence act as bottlenecks in terms of computational time. Code packages for multiple species solving a set of equations serve as an example. While some species may be treated in parallel at every timestep, there might exist inter-dependency among other species preventing parallelization and thereby eventually becoming bottlenecks in the parallelization process. This acts as a motivation to explore another dimension for parallelization which is time. Moreover, it must be clarified that temporal parallelization is not a replacement for any existing form of parallelization, but may be added to them to further, often significantly, improve performance. Temporal parallelization using the parareal algorithm was originally proposed in 1] in 2001, and has been applied to a variety of problems from relatively simple ones $[2,3,4,5,6,7,8$, to ones with complex dynamics [9, 10, 11, 12].

This work explores the application of the parareal algorithm in an area in fusion plasma. Simulations involving the edge and divertor regions of fusion devices are extremely challenging due to their complex physics as well as requirements for large wall-clock times. These computations involving the plasma edge are important for modeling plasma surface interactions along with designing the wall of fusion devices. The dynamics is complex at the scrape-off layer (SOL) which is the region between the device wall and the Last Closed Flux Surface (LCFS). Impurities, which may originate primarily from plasma interactions with the materials of the wall, may greatly dilute the plasma and negatively affect the plasma heating. The presence of impurities is also necessary, to some extent, for radiative cooling of the divertor region to mitigate the very high heat 
and particle fluxes onto the divertor targets, thereby achieving (partial) plasma detachment. A comprehensive modelling of the SOL is one of the greatest challenges of magnetically confined fusion plasmas. The SOL is characterized by open field lines. In addition to dominant parallel flows along these field lines, perpendicular flows become important in this region of the plasma. Also, alongside charged particle transport, neutral particle transport is dominant in some parts of the SOL. Neutral particles are externally introduced into the plasma through neutral beam injection, gas puffs and outgassing and removed by pumping. Processes within the plasma like recycling, sputtering and volume recombination are also hugely responsible for the presence of neutrals at the plasma edge of a fusion device [13, 14, and processes like ionization contribute to reducing their number. A range of codes, such as BOUT[15, 16], UEDGE[17], DIVIMP [18], ERO-JET [19], ASCOT[20], Edge-SOL[21], SOLPS [22, 23], EMC3Eirene 24] and EDGE2D-Nimbus 25], SOLEDGE-2D 26] with varied degrees of complexity have been used to study different aspects of the plasma edge. Incorporating optimum physics into these simulations is often a challenge due to the computational cost. With simulations targeting more ITER-like plasmas, the wallclock time may often exceed weeks or months. An example of such cases are studies involving particle-balance in the simulations [27, 28, 29].

Exploring the advantages of the parareal algorithm in edge plasma simulations is different in many ways from previous applications. Fully developed turbulence simulations in the steady state where the statistical study of properties such as the probability distribution function, vorticity and velocity are relevant are dealt with in [9, 10]. Although such an application is challenging due to the very high Reynolds number and Lyapunov exponent, the individual solutions per simulation are less important than their statistical behaviour. The case investigated in [9] involved a single cold fluid representation for all charged ion species, thus neglecting any interaction between them. Moreover, from the viewpoint of fusion plasma simulations, this was a case dealing with core-plasma turbulence, where there were no neutral species.

[11] sees the application of the parareal algorithm to the Corsica code which 
is a scenario modelling tool for tokamak plasmas. It simulates burning plasma scenarios but the details of the physics of the edge involving impurities and neutral transport are not given significant importance. Corsica is by itself a complex workhorse combining various codes/modules to compute different plasma dynamics such as equilibrium, transport, etc. However, the simulations are restricted within the separatrix or the last closed flux surface allowing one to neglect parallel transport. The Grad-Shafranov equation is solved to compute the MHD equilibrium. In simpler terms, Corsica models the (almost) whole of the plasma using simplified models for individual dynamics.

This work studies the application of the parareal algorithm to SOL simulations using the SOLPS-Eirene package [22. It is shown that if the algorithm is optimally applied, a speed-up of more than a factor of ten may be achieved for the cases studied here. The SOLPS-Eirene code package has been extensively used for existing tokamaks 22, 23, 29] as well as island divertor configurations [30, 31] and is also being used at ITER [29, 32. This application is significantly different from previous applications. SOLPS focuses on a relatively detailed study of a single region (edge) of the plasma. As already mentioned, the edge has open field lines, so transport is 2 dimensional (both parallel and radial). Also, the impact of the presence of neutral particles in this region of the plasma makes these computations more challenging and complex. The application to the SOLPS-Eirene package is unique and promising as it allows the possibility of applications to core-edge coupled codes for efficient simulations of ITER-like plasmas. The parareal implementation was performed using the IPS framework developed at ORNL, USA, as part of the SWIM project [33, 34]. The framework allows the use of 'event-based parareal' 35. which significantly improves performance and is discussed in more detail in sections 2.3 and 3.4 .

This paper is organized as follows. Section 2 describes the parareal algorithm and the techniques employed for its application. Section 2.1 gives a brief summary of the model employed for the parareal application. The results are described in section 3 and the findings are summarized in section 4 


\section{Parareal algorithm}

With increasing demands for reducing wallclock time and improving the scaling of computational gains with cores on modern supercomputers, parallelization of simulations has gained tremendous importance. Spatial parallelization is a common approach although the technique often reaches saturation preventing optimum resource usage. Exploiting the time domain allows one to utilize more cores and further speed up the code. Temporal parallelization is non-intuitive as it apparently violates causality. However, a number of predictor-corrector approaches to achieve time-parallelization (PITA [36], PFASST [37, parareal [1]) have been proposed which have garnered increasing attention in recent years. There has also been some work on combining time parallelization with space parallelization [38, 39]. The technique of the parareal algorithm has been discussed in detail in [1, 9. It involves dividing a time series into slices which are solved in parallel. This is illustrated in fig. 11. where individual time slices may be considered as $t_{0}$ to $t_{1}, t_{1}$ to $t_{2}, t_{2}$ to $t_{3}$ and so on. Each individual time slice is solved in parallel by different processors represented by P0, P1, P2, etc in the figure. The algorithm requires a coarse predictor $(\mathrm{G})$ which is computationally fast but gives only a coarse estimate of the solution. The fine (F) solver or the corrector is computationally slower but generates a solution with a higher accuracy. A parareal iteration is represented by $\mathrm{k}$ each of which comprises of a $\mathrm{G}$ and $\mathrm{F}$ run. The coarse solver is always applied serially across processors, while the fine computation is performed in parallel, leading to a computational speed-up. At $k=1, \mathrm{G}$ is applied to give each processor an initial value. Then, $\mathrm{F}$ is applied to that (wrong) initial value in parallel across every time slice. At the start of each subsequent parareal iteration, $\mathrm{k}$, the parareal correction is applied to the initial value across each processor (or time slice, i) and this correction is given by eq. 1

$$
\lambda_{i+1}^{k}=F\left(\lambda_{i}^{k-1}\right)+G\left(\lambda_{i}^{k}\right)-G\left(\lambda_{i}^{k-1}\right)
$$

$\lambda_{i+1}^{k}$ is the initial state for the $(\mathrm{i}+1)^{t h}$ time slice at the $\mathrm{k}^{\text {th }}$ iteration. At 
the $\mathrm{k}^{\text {th }}$ iteration $\lambda_{i}^{k}$ is evolved to $\lambda_{i+1}^{k}$ using $\mathrm{F}\left(\lambda_{i}^{k}\right)$ and $\mathrm{G}\left(\lambda_{i}^{k}\right)$.

The coarse and fine calculations are repeated across a number of parareal iterations until convergence is achieved. Parareal convergence across a processor is said to have been achieved when the 'defect' in two successive fine calculations ( $k$ and $k-1)$ across that processor is below a tolerance value. The sum of this defect in solutions across a time chunk between $t_{i}$ and $t_{i-1}$ is defined by

$$
\zeta_{i}^{k}=\int_{t_{i-1}}^{t_{i}} \frac{\left|\lambda^{k}(t)-\lambda^{k-1}(t)\right|}{\left|\lambda^{k-1}(t)\right|} d t .
$$

The solution is then converged for time slice $i$ if,

$$
\zeta_{i}^{k}<\text { tol, } \forall i \leq I
$$

\subsection{SOLPS-Eirene code package}

The interactions between the plasma and the wall in tokamaks and other fusion devices play an important role in the success of the experiment. The region of interest for this purpose is known as the scrape-off layer or SOL. The SOL is bounded by the wall of the machine and the last closed flux surface as shown in fig, 2 The charged particles travel along the field lines and reach the divertor or target plates. A variety of configurations may be possible, two of which have been explored in this work. Depending on the number of null points or x-points in the magnetic field, a tokamak may have single, double or multiple (snowflake) null geometry. One of the tokamaks explored in this work for parareal simulations in the SOL is MAST with double null geometry and two sets of upper and lower divertors, illustrated in fig.2 DIII-D is the other fusion device simulated here and it has single null geometry and only lower divertors. The purpose of this paper was demonstrating the effectiveness of the parareal algorithm in simulations of two separate machines thus paving the way to broadening of the areas of application. In the SOL, the plasma may be treated as a fluid while the neutral particles may be simulated as Monte-Carlo histories. The SOLPS (Scrape Off Layer Plasma Simulator) code package consists of the 2D multifluid transport code B2.5 coupled with a neutrals transport model. The 


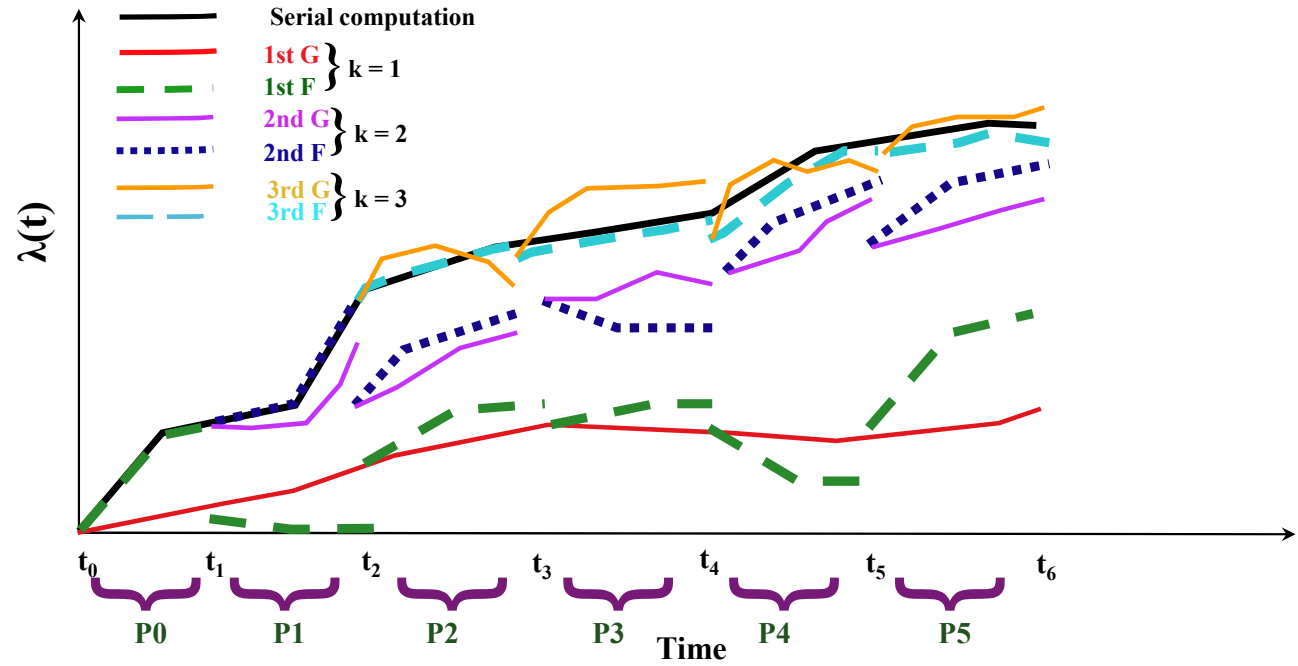

Figure 1: The parareal algorithm involves a coarse $(G)$ and a fine $(F)$ solver. The combination of a single $\mathrm{F}$ and a single $\mathrm{G}$ computation constitutes each parareal iteration, $\mathrm{k}$. The simulation starts with the $1^{\text {st }} \mathrm{G}$ which is a sequential process. This is followed by the $1^{\text {st }} \mathrm{F}$. F is solved in parallel over multiple time slices $\left(t_{0}-t_{1}, t_{1}-t_{2}, \ldots, t_{n-1}-t_{n}\right)$. Each time slice is solved by individual processors $P_{0}, P_{1}, \ldots, P_{n}$. After several iterations or $\mathrm{k}$, the parareal solution approaches that achieved by serial computation (given in black). 
B2.5 model is based on the Braginskii equations [40] in magnetically aligned curvilinear orthogonal coordinates. The set of equations include the continuity and momentum conservation equations for each ion species, current continuity equation and the energy balance equations for both ions and electrons, which are described in detail in 22,41$]$. Electron density is determined via quasineutrality. In the cases explored in this work, the $\mathbf{E} \times \mathbf{B}$ and diamagnetic drifts 42 ] are absent.

The transport of neutrals is modelled using the Eirene package 23]. Eirene uses Monte-Carlo treatment of neutral particle transport solving the Boltzman equation for distribution functions for neutrals. The kinetic model is detailed in physics and computationally robust but is expensive in terms of wall-clock time. In fact, Eirene is often responsible for significantly slowing SOLPS simulations.

\subsection{Coarse solvers for SOLPS}

As has been discussed in [9, 10, 11, a variety of choices may be employed to achieve shorter wallclock time accompanied by coarseness in the solution. However, the choice is certainly non trivial. A coarse solver that is computationally fast may actually deviate the solution very far away from the fine computation, which may lead to a high number of parareal iterations to achieve convergence or convergence may not be achieved at all. On the other hand, the solver may be relatively slower, but the solution may be corrected quickly to approach the fine solution requiring fewer parareal iterations. The condition of the 'coarseness' of the fast solver has been outlined in [1, but it is almost impossible to translate that for a complex physics problem.

Two coarse solvers or $\mathrm{G}$ of the solution have been explored in this work for various implementations of the parareal algorithm to the SOLPS code package. As already mentioned in section 2.1 the fine or $\mathrm{F}$ version of SOLPS utilizes B2.5 along with Eirene for calculating the neutral transport in the plasma. Since Eirene is computationally expensive, replacing it by a fluid neutrals model simplifies the computation. As an example, the source term, $S^{n}$ in the continuity equation in its most general form given by eq 4 is computed differently in the 
two models.

$$
\frac{\partial n}{\partial t}+\nabla \cdot f l \vec{u} x=S^{n}
$$

The reduced model as well as the Monte-Carlo technique are discussed in detail in 22, 43, 44, 45]. Modifying the transport coefficients (and eventually boundary conditions) for the neutrals (and ion energy equation) to improve the coarse estimate reduced the number of parareal iterations required for convergence. The results of using the neutral fluid model in place of Eirene are discussed in section 3.1 . The second coarse solver is implemented using a coarse mesh yielding very interesting results. The technique of converting between coarse and fine grids is discussed in detail in 46. It has been observed that the Monte-Carlo method in the Eirene code for computing the physics of the neutral particles imposes a limitation on the size of the time step [23, 29, 47]. Using a coarser mesh in space increases the number of Monte-Carlo particles

per cell, thereby reducing the noise. This allows one to take bigger timestep sizes and still achieve the desired numerical accuracy and is very useful in significantly speeding up the computation. The results are discussed in section 3.2 The two coarse solvers studied in this work are independently implemented. A combination of the two is likely to yield the most computational gain.

\subsection{Event-based parareal with IPS framework}

The parareal implementation of SOLPS was achieved using MPI parallelization via the IPS (Integrated Plasma Simulator) framework. The IPS framework written in Python [33, 34, 35] launches an event-based parareal application using MPI. In the traditional, sequential technique, for every parareal iteration $\mathrm{k}$, all processors need to undergo 'mpi-wait' until computations for every time slice is completed for a given $\mathrm{k}$. This significantly increases wall-clock time. This room for improvement was originally addressed in all implementations such as pipelined 48, scheduled [4] and event-based. The event based framework further extends the concepts of [48] and [49] by exploiting a data driven work-flow 
where processors are assigned tasks that are independent of a previously computed task by the same processor. This maximizes resource utilization thereby significantly improving the computational gain. This feature is described in detail in [33, 34, 35].

The 'event-based parallelism' makes optimum use of resources possible. Here, for any iteration $\mathrm{k}$, if the coarse calculation has been completed for a time slice, $i=\alpha$ (say), the fine computation may be done on all time slices with $i \leq \alpha$, while the coarse calculation may be carried out simultaneously on slices $i>\alpha$. The positive impact of the event-based approach on the parareal gain and efficiency is discussed in section 3.4. Moreover, the IPS framework simplifies the implementation of the algorithm in comparison to a traditional MPI approach to any code. Unlike a standard MPI application which involves introducing significant changes to the original serial code, there was no rewriting of the underlying physics codes in case of the IPS framework.

\section{Results}

With the purpose of exploiting the parareal algorithm in edge plasma simulations, our studies involved two tokamaks with different geometries. MAST is a spherical tokamak with double null geometry as described in fig. 2 The other machine used as a testbed for our simulations is DIII-D, which has a single $\mathrm{x}$-point and hence single null geometry. Fig. 3 shows the fine mesh for SOLPS parareal simulations which is the same as the one used for serial computations.

The cases explored in this work had low density at the targets and the temperature of the plasma at the targets were $(\sim 40 \mathrm{eV})$ in MAST [41] and $(\sim$ $10 \mathrm{eV}$ ) in DIII-D. For both machines, the plasma species consisted of Deuterium and Carbon ions. The primary variables of the code are ion density (na, $m^{-3}$ ), electron density $\left(\mathrm{ne}, \mathrm{m}^{-3}\right)$, electron (te, $\mathrm{eV}$ ) and ion temperatures (ti, eV), parallel velocity $\left(u_{\|}, m / s\right)$ and electrostatic potential $(\phi, V)$. The parareal correction, given by eq1 1 was applied to these six variables at the beginning of a time slice for a given parareal iteration, k. The maximum total power 


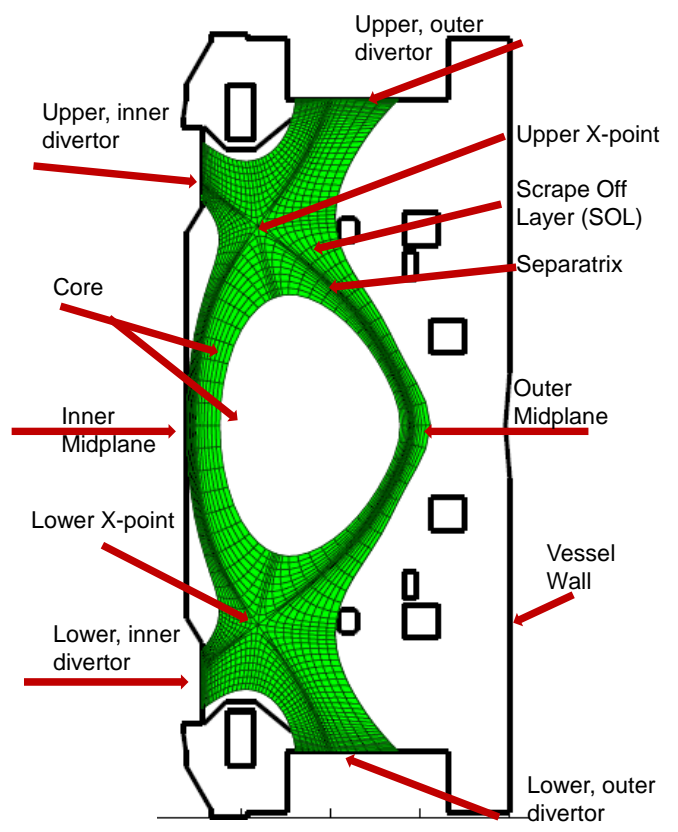

Figure 2: The mesh used for SOLPS simulations of MAST. 


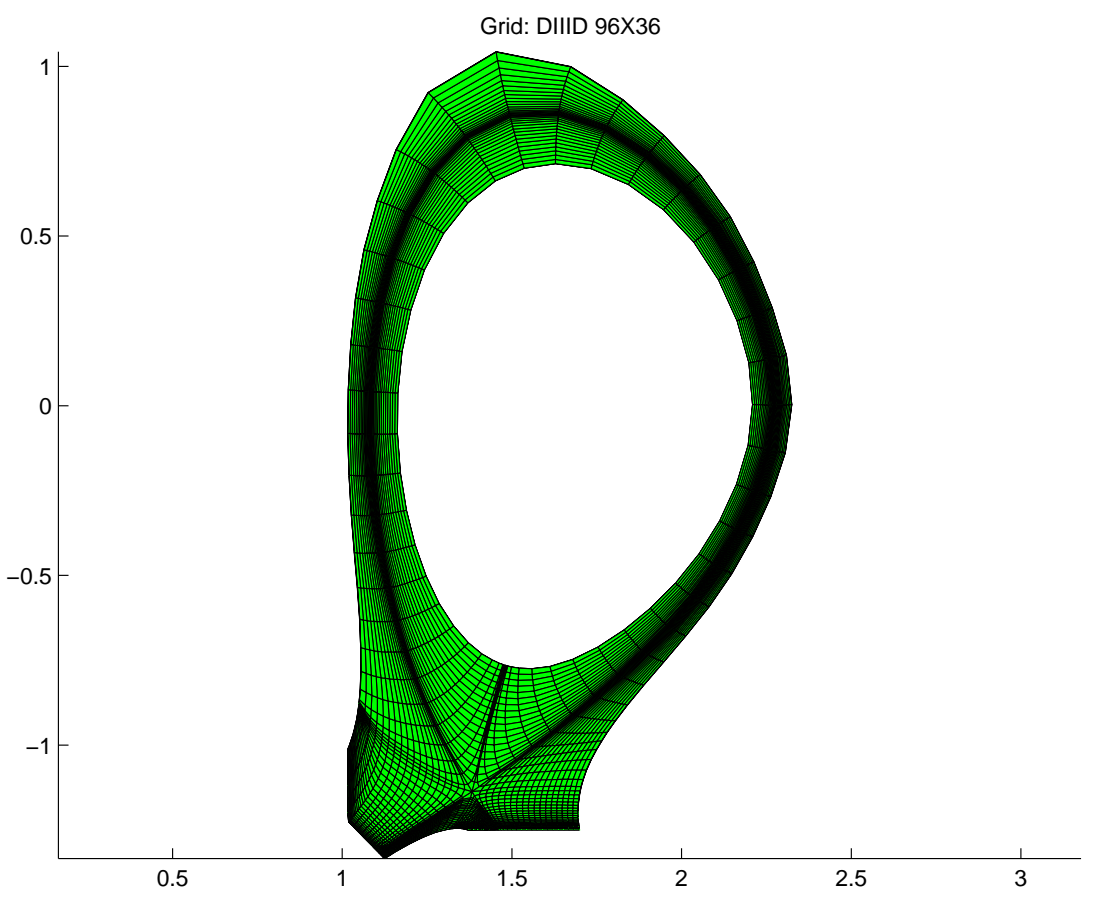

Figure 3: The fine grid layout of the DIII-D tokamak used for SOLPS simulations. This grid of size $96 \times 36$ was used for serial computations as well as the 'fine' or F run of the parareal implementation. 
fluxes $\left(W / m^{2}\right)$, pwmxip and pwmxap, on the inboard and outboard divertor respectively were chosen as convergence metrics and are represented by $\lambda$ in eq. 2 . After eq 2 is applied to both these variables, convergence at a given iteration $\mathrm{k}$ is said to be achieved when the defects for both these variables satisfy eq3. The choice of tol in eq 3 was based on the values of the residuals of the equations solved. The SOLPS code package implicitly solves every equation leaving a small residual. The attempt was made to ensure that the parareal solution had the same residual as compared to a serial (fine, F) solution. A series of simulations with test cases were run for this purpose. One such case, where the evolution of the values of the normalized residuals for the parareal solution gradually approaching the serial value, is demonstrated in fig 4 Fig 4 shows that the values of the normalized residuals decrease with increasing parareal iterations, $\mathrm{k}$ and at $k=8$ approach the values as obtained in the fine (F) simulation (fig 5 ). It was observed that when tol in eq 3 was $5 \times 10^{-2}$, the residuals of the parareal solution were in acceptable agreement with those of the serial or fine run, which is demonstrated in fig 5 . So, in the simulations, parareal convergence is said to have been achieved when the defect in successive solutions, $\zeta$ for both pwmxip and pwmxap are $\leq 5 \times 10^{-2}$. Using a metric as in eq 2 allows continuity across various time slices which is often a cause of concern in parareal simulations. However, incorporating its dependence on the residuals of the solutions also ensures a steady solution.

As has been mentioned in section 2, the parareal algorithm divides the entire time domain into multiple time slices. We define each time slice as the physical time (in seconds) simulated by each processor and denote it as $t_{\text {slice }}$. For numerical time-stepping, each $t_{\text {slice }}$ is divided into ntim timesteps, and each timestep is of size dt seconds. So, for clarity, it may be stated that $t_{\text {slice }}=n t i m \cdot d t$. In the parareal implementation, each time slice is simulated by an individual processor. Therefore, if there are $\mathrm{N}$ processors, the total physical time that is simulated is $\left(N \cdot t_{\text {slice }}\right)$ seconds. It may also be noted that the size of a time slice may be varied by varying either or both of ntim and $\mathrm{dt}$.

Since each time slice is discretized, the values of pwmxip and pwmxap are 


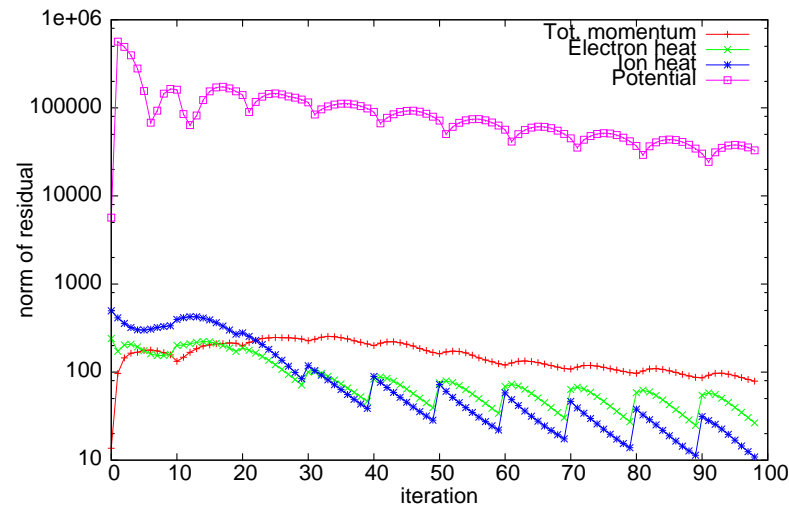

(a) Parareal iteration, $k=1$.

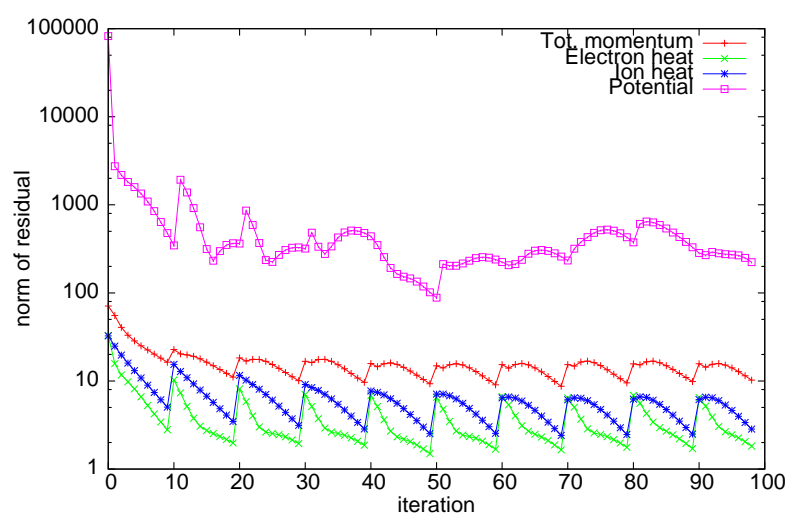

(c) Parareal iteration, $k=6$.

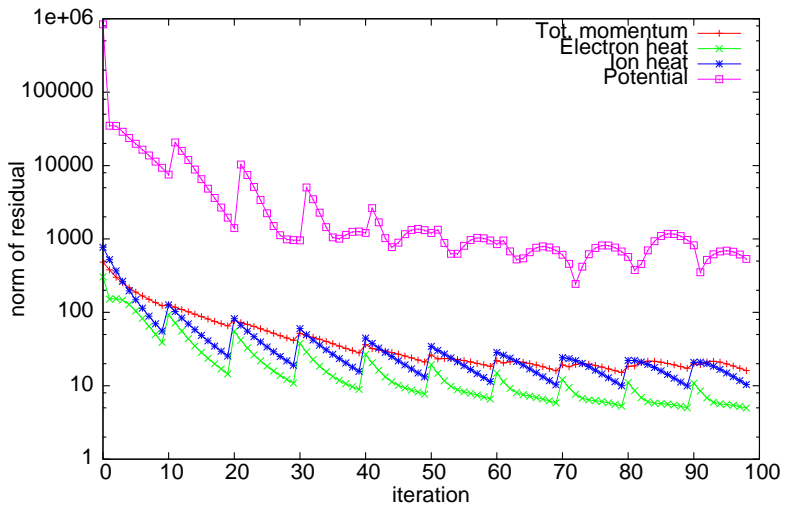

(b) Parareal iteration, $k=3$.

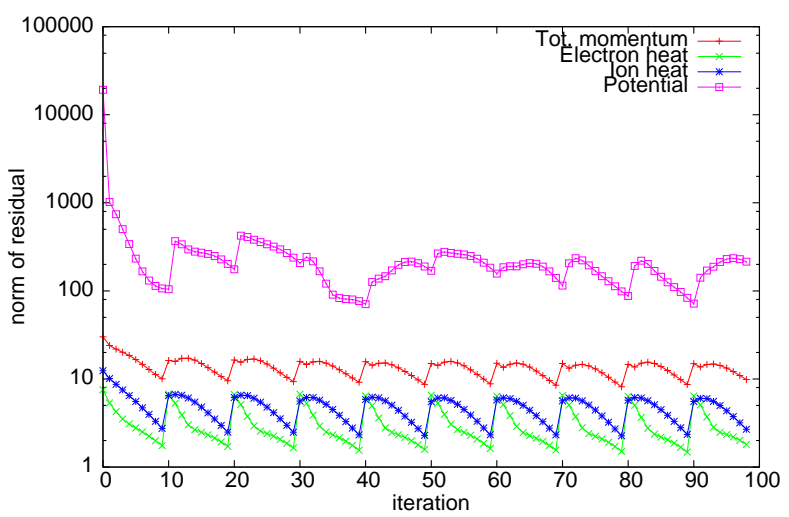

(d) Parareal iteration, $k=8$.

Figure 4: The norm of the residuals for equations solved across a given time slice decrease with increasing parareal iterations, $\mathrm{k}$. 


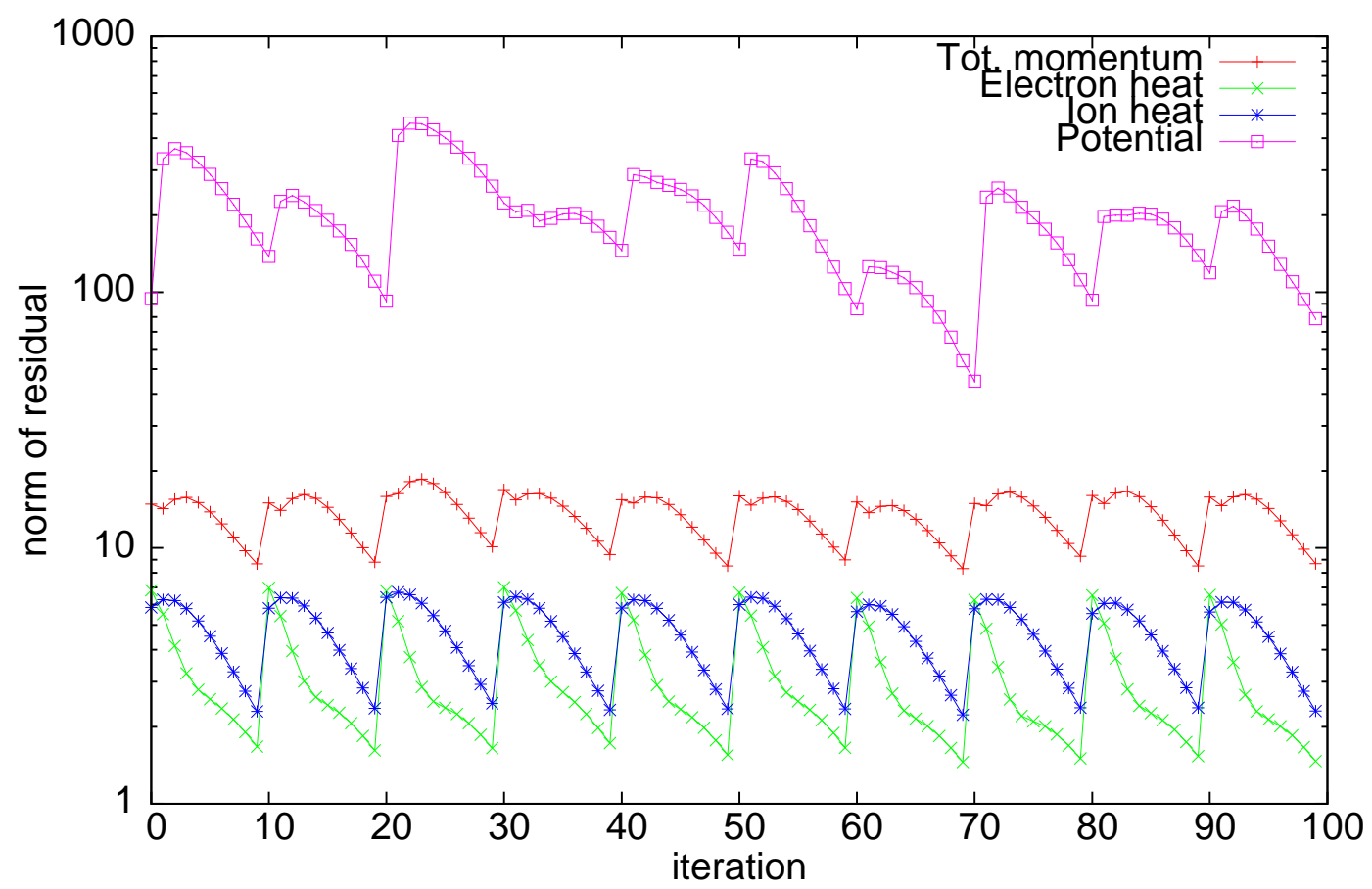

Figure 5: The norm of the residuals for the equations as computed by the SOLPS code package for a serial or fine $(\mathrm{F})$ simulation. The tolerance of the parareal computation is chosen in such a way so that the residual for the parareal calculation agrees with those of the fine $(\mathrm{F})$ simulation. 
stored at the end of each time step of size dt. Therefore, at the end of a given parareal iteration $\mathrm{k}$, the number of values of each of pwmxip and pwmxap that are stored for a processor is equal to ntim. These ntim values are then used to compute the integral in eq.1.

It must be noted that all computational gain is calculated with respect to the wallclock time of a serial run, which in this case is the fine (or, F) run. With the fine and parareal run times given by $T_{F}$ and $T_{P R}$ respectively, over the same simulation length $\left(N \cdot t_{\text {slice }}\right)$, the computational gain is defined as

$$
\text { gain }=\frac{T_{F}}{T_{P R}}
$$

\subsection{Coarse solver using neutral fluid model}

Replacing the kinetic model in Eirene with the neutral fluid model speeds the simulation by approximately 49 times but the accuracy of the solution is compromised. For example, the outer (nesepm1) and inner (nesepm2) midplane separatrix densities for the electrons are shown in figs. 6a and 6b where the final desired fine computation is significantly different from the initial coarse estimate. This coarse predictor with simplified physics was used in simulations of MAST. For every time slice solved, the time-step size (dt), and hence the number of timesteps (ntim), were the same for both the fine and coarse runs.

Using the case of fig 6 as a coarse predictor, the fine solution may be recovered in much shorter wall-clock time using the parareal algorithm. With increasing parareal iterations, $\mathrm{k}$, the solution approaches the value that is typically obtained using a serial computation with the fine solver (as in fig 6a. The evolution of the parareal solution for the density at the separatrix (nesepm) across iterations is illustrated in fig,7, Figs. 8a, 8b, 9 demonstrate that the parareal solution (in this case, the maximum total power flux at the outer divertor) is identical to the serial solution, despite starting the simulation with the coarse computation in fig. 8a. Since MAST has two sets of divertors (upper and lower) resulting from the double null configuration shown in fig. 2, in the 


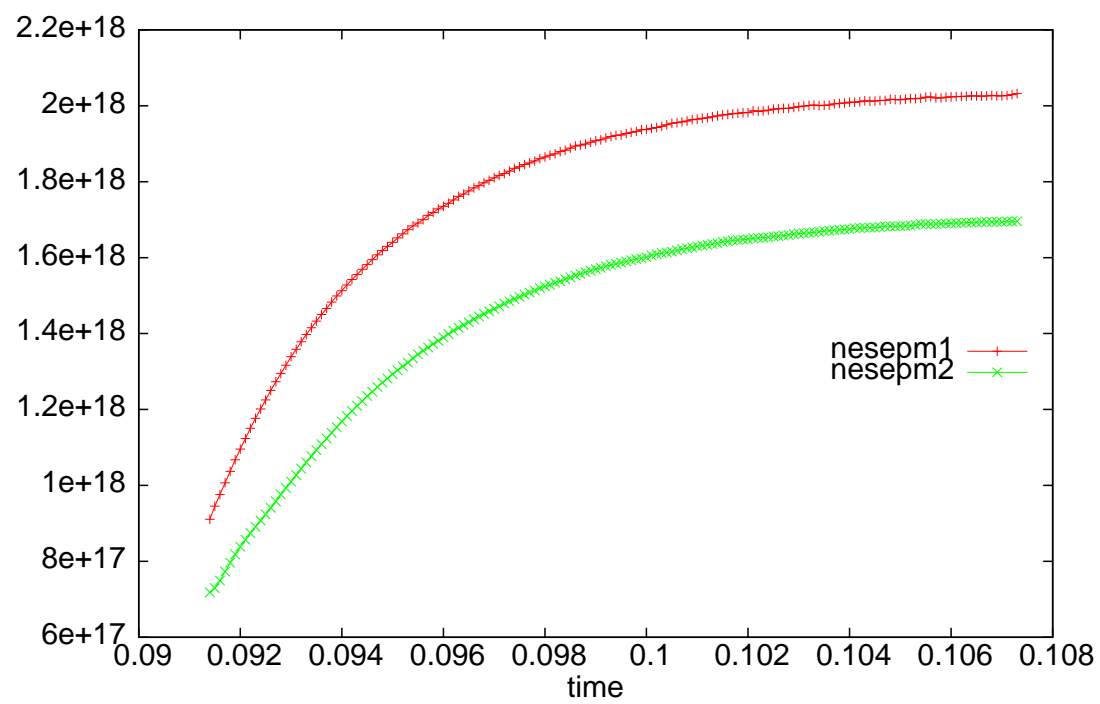

(a) Fine computation using the SOLPS-Eirene package.

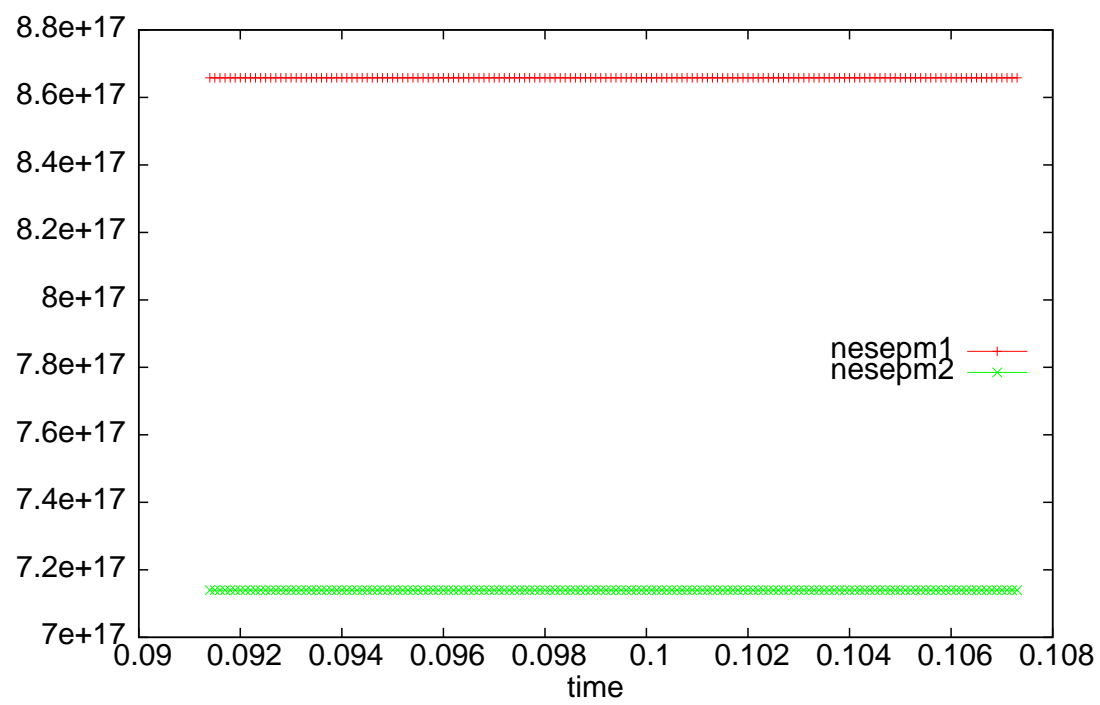

(b) Coarse solution (Eirene replaced by fluid neutral model).

Figure 6: The midplane density (nesepm1 for outer and nesepm2 for inner) varies when computed by the fine $(\mathrm{F})$ and coarse $(\mathrm{G})$ propagators. 


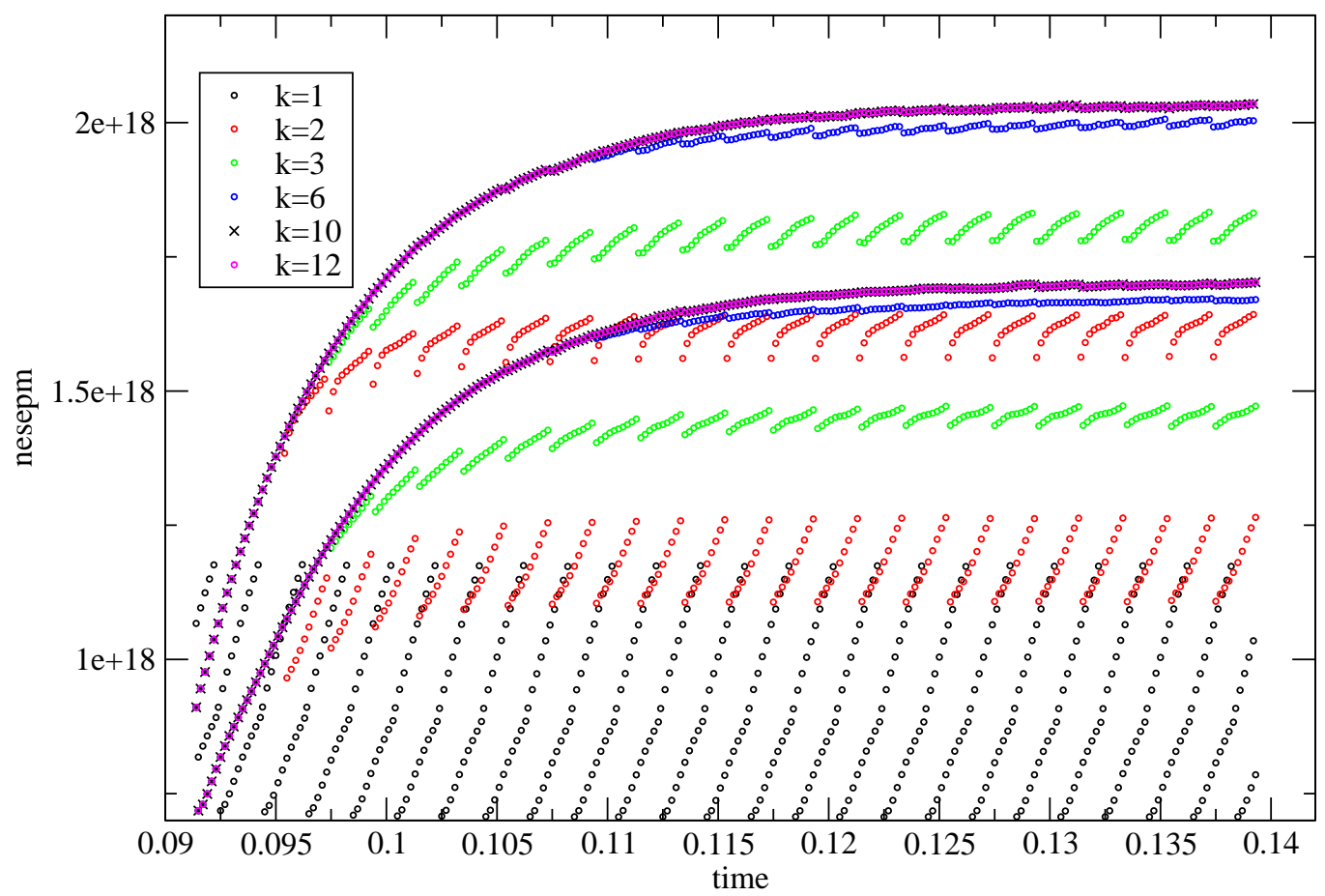

Figure 7: The plot of density at the separatrix plotted for various parareal iterations, k. At the final iteration, $k=12$, the solution matches that of the fine solver in fig $6 \mathrm{a}$

plots figs. 8a, 8b, 9, pwmxap 1 refers to the lower outer divertor target while pwmxap $_{2}$ represents the value at the upper outer divertor target.

A gain of 12.58 was obtained with 240 processors for the case just discussed, where each processor solved a time slice consisting of number of time-steps, $n t i m=10$, with $d t=1 E-4$. Convergence was achieved after $k=12$ parareal iterations in this case. It was interesting to explore if the size of the time slice, i.e, $t_{\text {slice }}$ had an impact on the parareal convergence. For this purpose, dt was kept constant and the number of time-steps per slice, ntim was varied. It was observed that $n$ tim $=10$ was optimum. Parareal convergence was not attained 


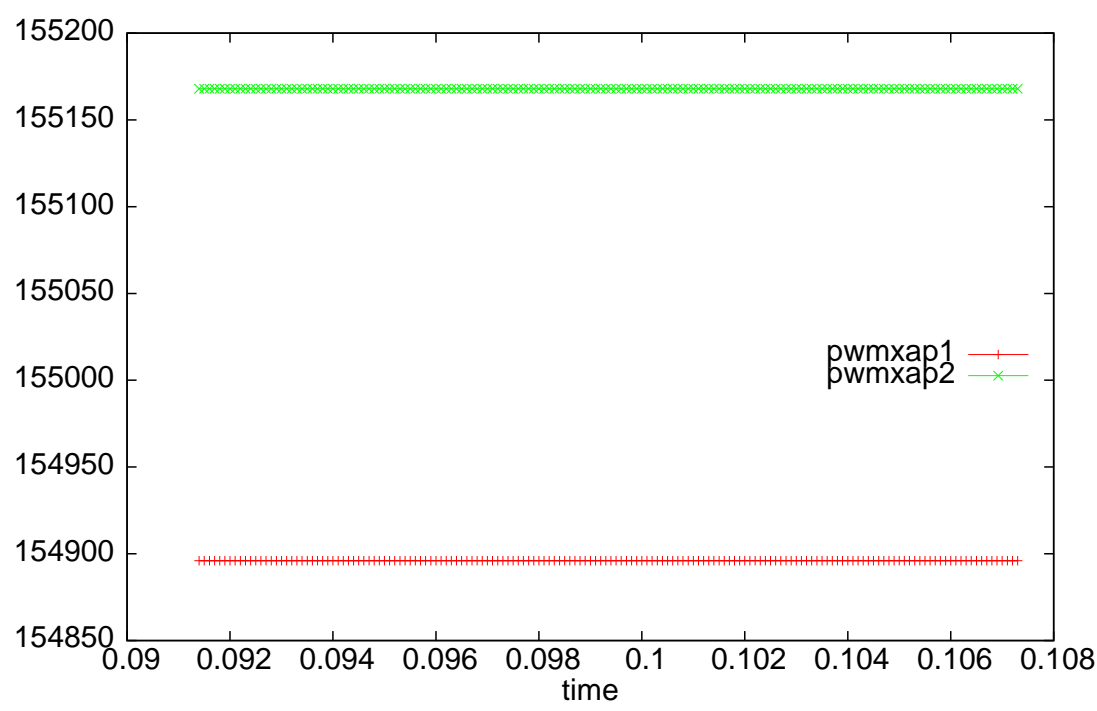

(a) Coarse (G) solution.

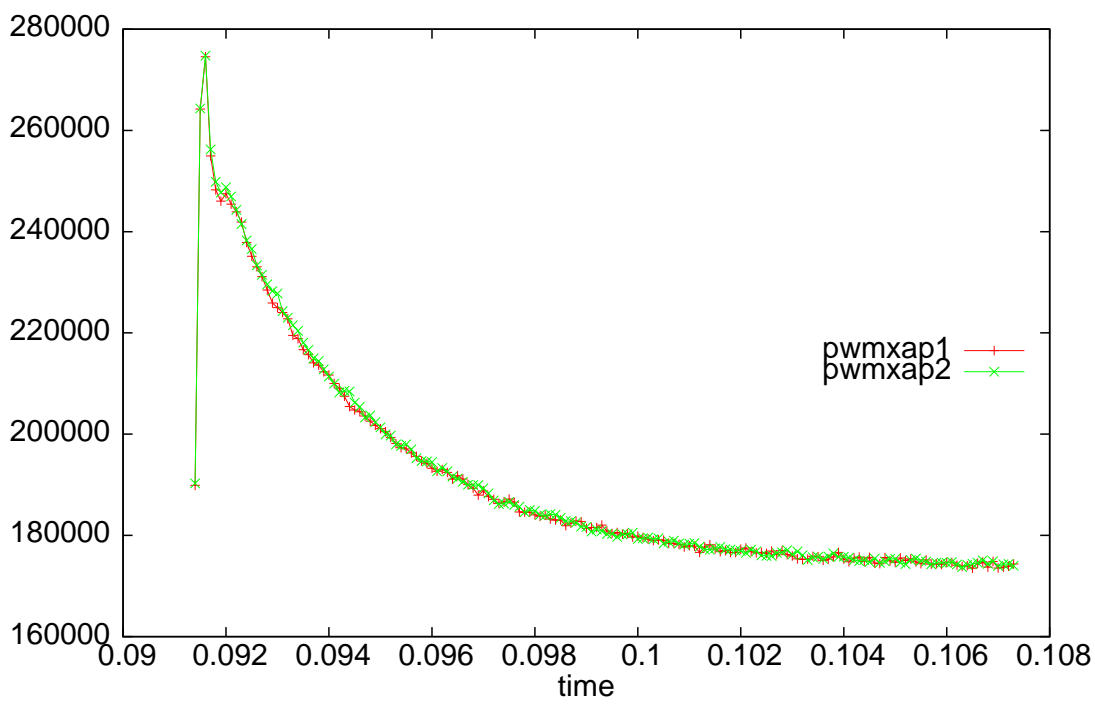

(b) Fine (F) solution.

Figure 8: Maximum total power flux are given by pwmxap $_{1}$ (lower) and pwmxap $_{2}$ (upper) at the outer divertors. A fluid model was used in the coarse $(G)$ computation for the transport of neutral particles while a Monte-Carlo method was used for the fine (F). 


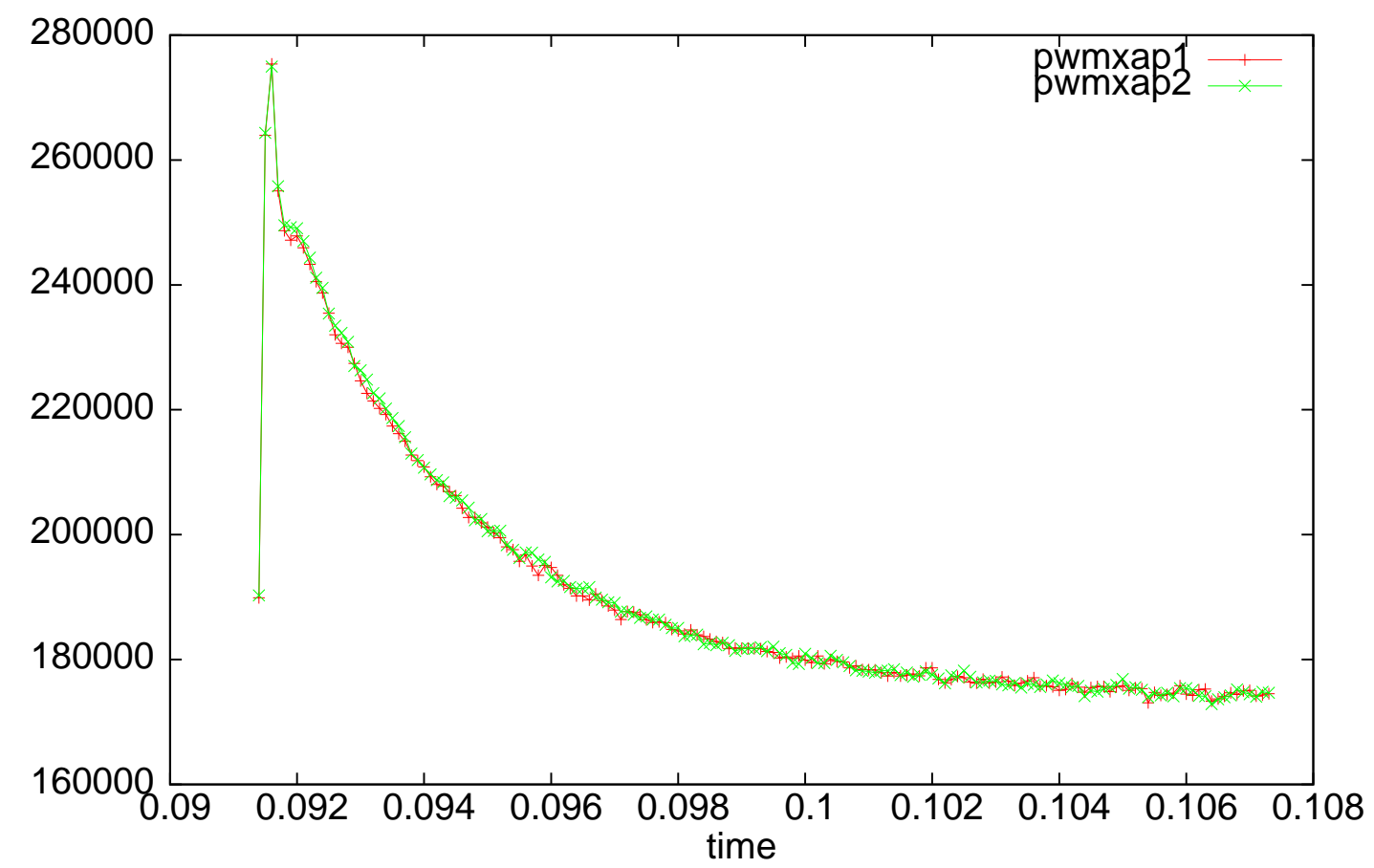

Figure 9: The final parareal solution matches the serial solution showin in fig $8 \mathrm{~b}$ pwmxap and pwmxap $_{2}$ are the maximum total power flux at the lower and upper outer divertors respectively. 
when the number of time-steps solved on each time slice was $\leq 5$ or $\geq 40$. For slices with ntim $\leq 5$, it is likely that the simulation per processor was not long enough to attain the fine values before it was restarted with a new initial value for the next iteration. This is illustrated in fig 10 where even after $k=11$ parareal iterations, the defect in solutions at $k=11$ and $k=10$ is not below $5 E-2$.

It was also observed that an unlimited increase of the size of the time slice solved per processor by increasing ntim may negatively impact convergence. This is likely because the coarse solution is allowed to deviate very far from the fine solution which essentially violates the condition for parareal correction to rectify the solution as formulated in [1]. A similar behaviour with parareal simulations has been observed and studied in [9, 10].

\subsection{Coarse solver using reduced grid and bigger timesteps}

Increasing the timesteps size or dt in the simulation allows faster computation but may introduce inaccuracy, as discussed in section 2.2. The second coarse solver explored in this work is using a reduced grid with bigger timesteps size for faster computations. The parareal implementation was carried out in simulations on both MAST and DIII-D cases. The grid size in a simulation is given by $n x \times n y$ where $n x$ is the mesh size in the poloidal direction and ny is that in the radial direction. The fine computation for MAST used a grid given by $150 \times 36$, while for DIID simulations, the grid was of size $96 \times 36$. Various coarse grids were explored for both cases. For coarse computations of MAST simulations, grids of size $150 \times 18,76 \times 36$ and $76 \times 18$ were used. Grids $48 \times 36$ and $48 \times 18$ were explored for the same purpose in DIII-D simulations. Using a reduced grid implies bigger spatial step sizes, which allows bigger time-steps (dt) without compromising on numerical inaccuracy. It must be noted, however, that the size of the time slice is same for both fine and coarse computations. Hence, if $d t_{F}$ and $d t_{G}$ are the sizes of the time-steps in coarse and fine computations respectively, for a given simulation,

$$
t_{\text {slice }}=N T I M_{G} \cdot d t_{G}=N T I M_{F} \cdot d t_{F}
$$




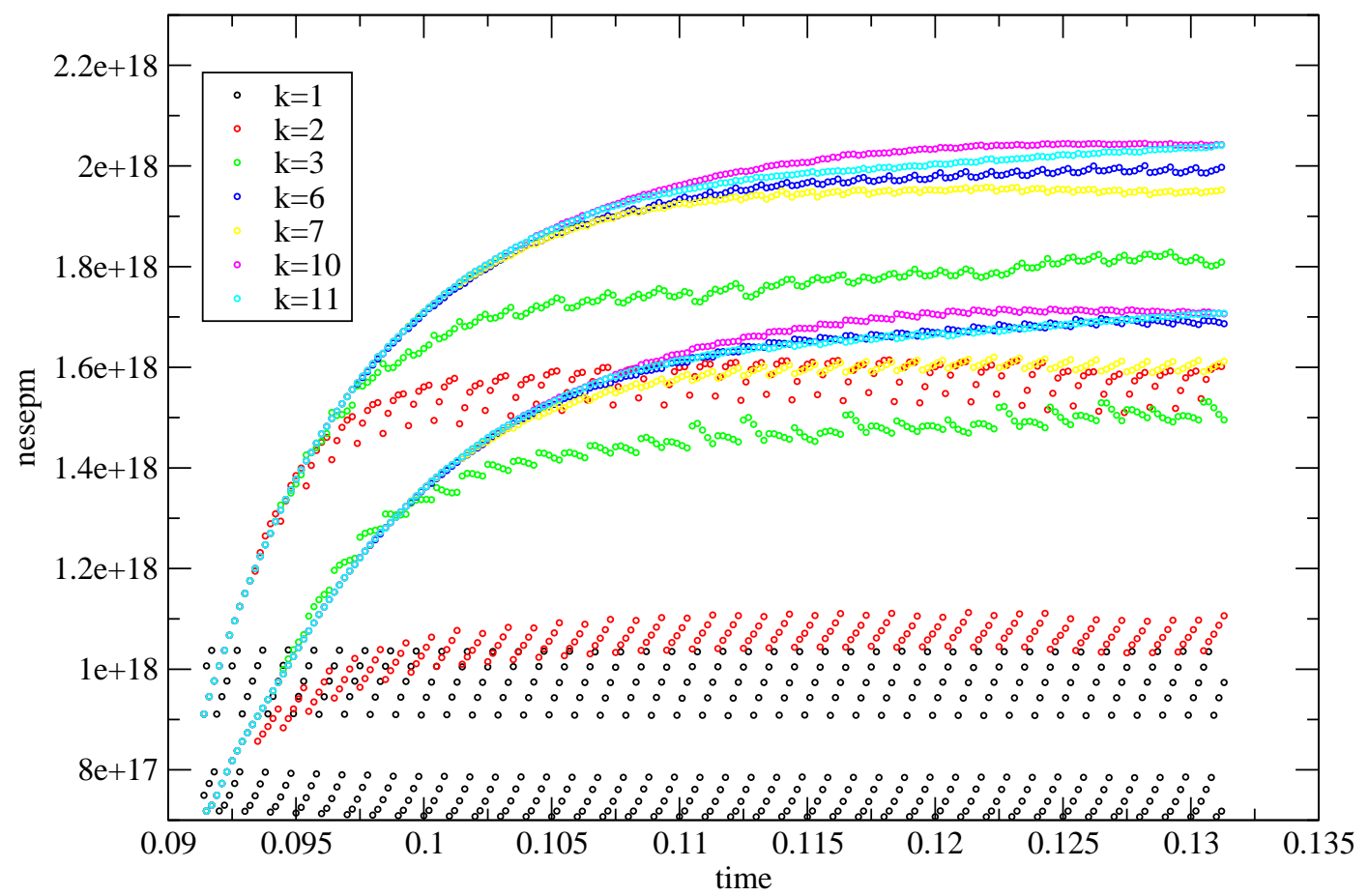

Figure 10: When the coarse solver used the fluid neutral model in place of Eirene, parareal convergence was not obtained when the size of the time slice per processor was $\leq 5$. The solutions never settle at a steady value even at large $\mathrm{k}$ (parareal iteration) until $k=i$ where $i$ is the processor number or time slice. 
The technique of using the reduced grid model for coarse $(\mathrm{G})$ computations yielded excellent results for both MAST and DIII-D studies. For DIII-D simulations, a coarse grid size of $48 \times 36$ and $d t_{G}=30 d t_{F}$ generated a computational gain (given by eq. 5) of 21.8 using 96 processors. A reduced grid of $150 \times 18$ and $d t_{G}=8 d t_{F}$ similarly yielded a parareal gain of 15.9 with 64 processors in MAST simulations. $d t_{F}$ and $d t_{G}$ are the fine $(\mathrm{F})$ and coarse $(\mathrm{G})$ time-steps respectively. The evolution of the solution in case of MAST with increasing parareal iteration $\mathrm{k}$ is illustrated as an example in fig.11. 'pwmxip' is the maximum total power flux impinging on the upper inboard divertor.

However, it was once again observed as in section 3.1 that the size of the time slice $\left(t_{\text {slice }}\right)$ strongly influenced the number of parareal iterations required for convergence. As a test case in DIII-D with 16 processors, fig. 12 illustrates this point. NTIMF and $N T I M_{G}$ are the number of timesteps solved on each processor for fine $(\mathrm{F})$ and coarse $(\mathrm{G})$ computations, respectively. It may be observed that there is a 'sweet spot' in the relation between $N T I M_{F}$ and $N T I M_{G}$ where the performance is best. Although the ratio of $N T I M_{F}: N T I M_{G}$ may stay constant, the computational gain changes depending on the exact values of $N T I M_{F}$ and $N T I M_{G}$. The dependence of the parareal iteration, $\mathrm{k}$ on the size of the time slice was studied for MAST simulations as is illustrated in fig. 13 . Here, the parareal iteration $\mathrm{k}$ required for convergence depended on $\operatorname{NTIM}_{F}$. $d t_{F}$ and $N T I M_{G}$ were kept constant, while $d t_{G}$ was varied along with $N T I M_{F}$ to satisfy eq. 6 .

Finally, a scaling study further supports the above points. DIII-D cases are used as examples for this case. For the weak scaling, the size of a time slice $\left(t_{\text {slice }}\right)$ per processor is fixed but the number of time slices are varied by varying the number of processors $(\mathrm{N})$. Hence, the total simulation length, given by $N \cdot t_{\text {slice }}$ varies with $\mathrm{N}$. The performance with weak scaling is demonstrated in fig. 14 with $N T I M_{F}=450$ and $N T I M_{G}=15$ per processor. $T_{F}$ in eq 5 increases with increasing simulation length, thus leading to the initial rise in gain in fig. 14 However, with increasing $\mathrm{N}$, the number of parareal iterations required for convergence also eventually increases leading to a flattening 


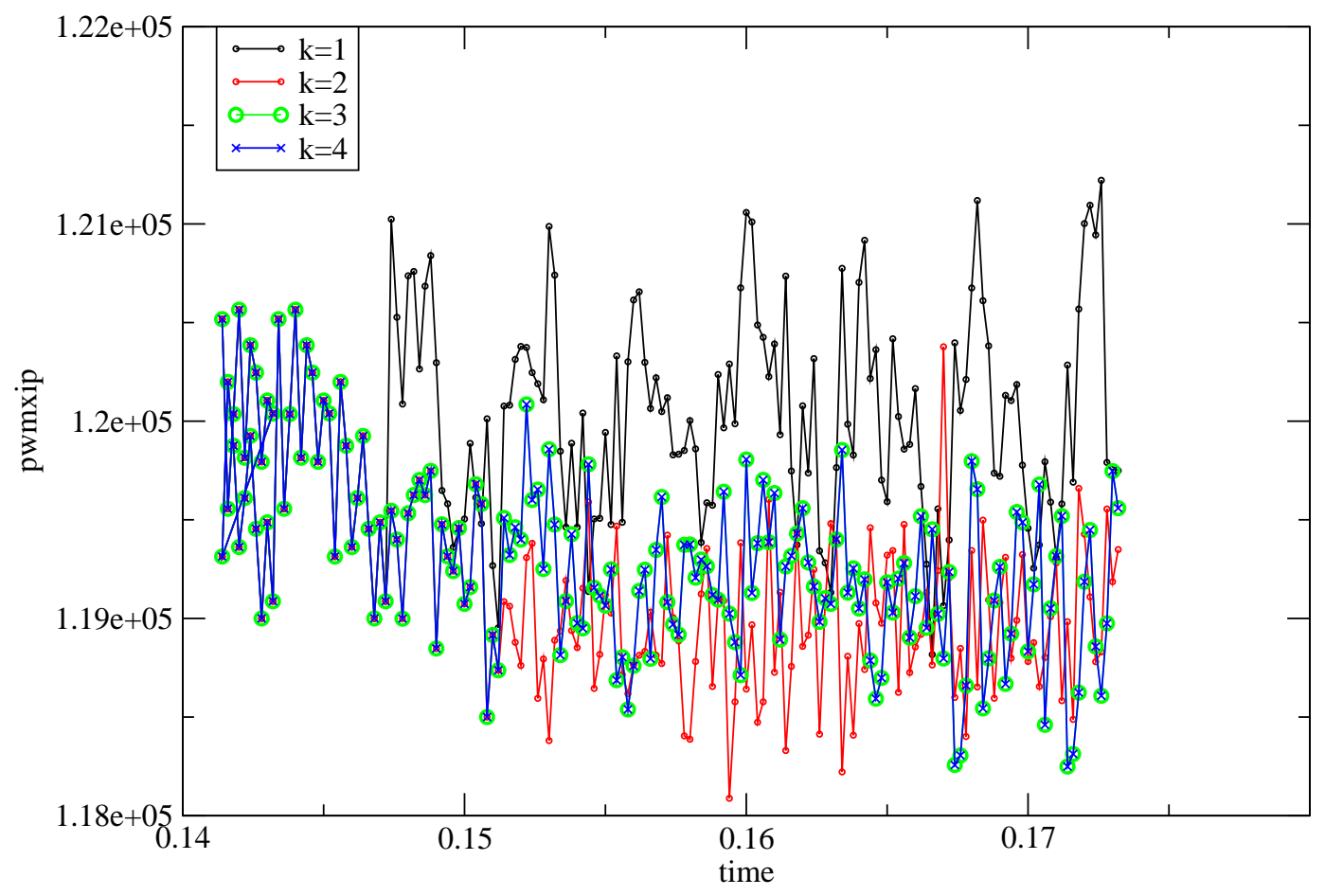

Figure 11: The parareal algorithm converges in 4 iterations for this case where the coarse model is a reduced grid. The defect in solutions (as defined in eq 2 for the total power fluxes at the inboard divertor for iterations $k=3$ and $k=4$ is below $5 E-2$. 


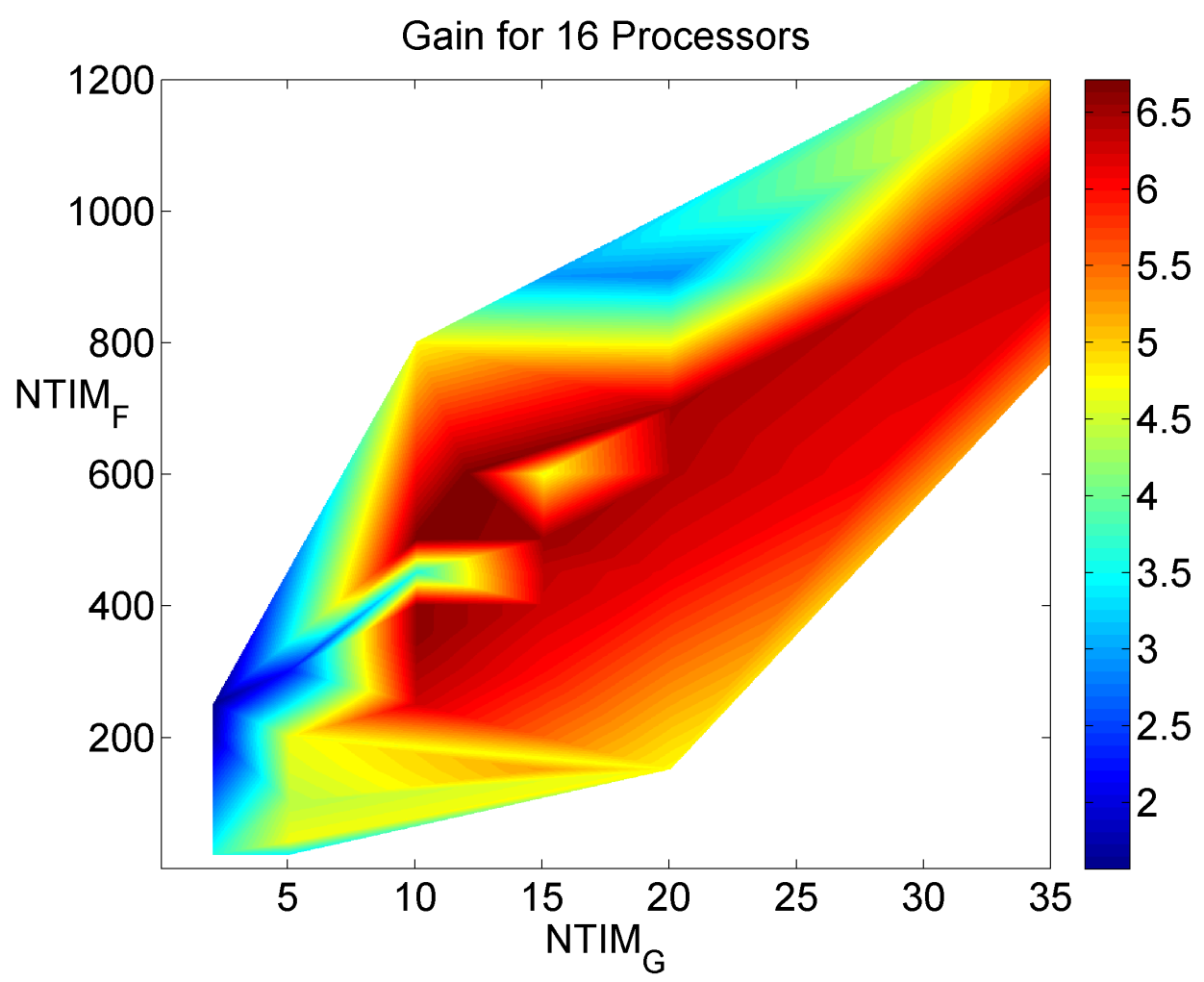

Figure 12: The parareal gain depends on the number of timesteps $\left(N T I M_{F}\right.$ for fine and $N T I M_{G}$ for coarse) solved per processor. 


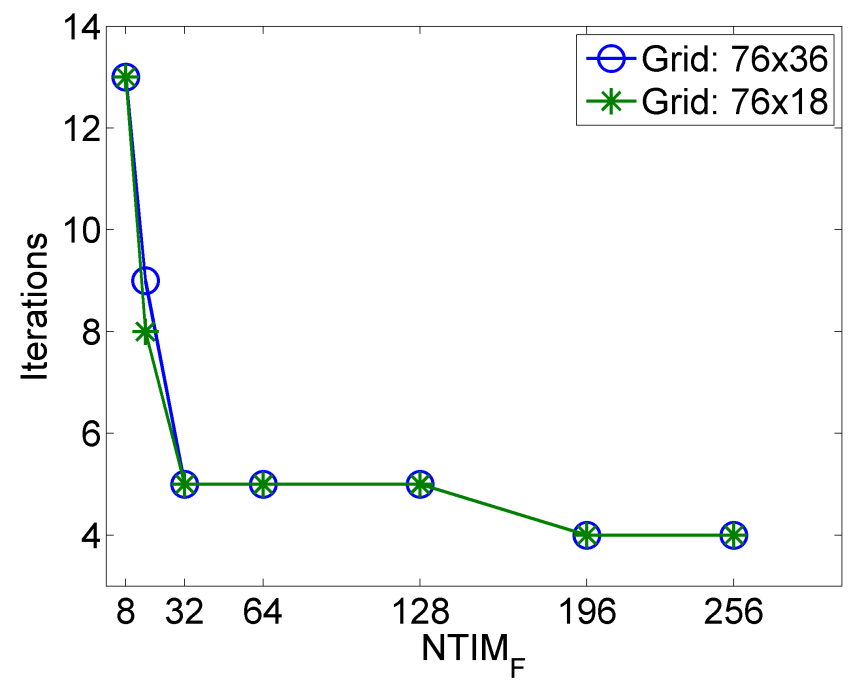

Figure 13: For the same number of timesteps solved per processor for the coarse computation, the parareal iteration $\mathrm{k}$ required for convergence depends on the number of timesteps used for the fine computation $\left(N T I M_{F}\right)$.

of the computational gain. The gain can always be maximized by optimizing $N T I M_{F} / N T I M_{G}$.

In case of strong scaling, the total simulation length is fixed while the number of processors $(\mathrm{N})$ is varied. $d t_{F}$ and $d t_{G}$ are also kept constant. Thus, both $N T I M_{F}$ and $N T I M_{G}$ vary with the number of processors, N. The strong scaling is shown in fig. 15 in a case where $\frac{N T I M_{F}}{N T I M_{G}}=12.5$. For high processor count $(\mathrm{N})$, the time slice solved per processor (and hence $N T I M_{F}$ and $N T I M_{G}$ ) is significantly reduced. The reduction in the values of $N T I M_{F}$ and $N T I M_{G}$ reduces the gain reflecting the observations in fig. 12 . It may also be noted that the performance using the coarse solver with reduced grid was more robust than the one in section 3.1 as a wider range of time slices were allowed where parareal convergence was achievable.

\subsection{Parallel efficiency and energy cost}

The simulations were performed on the Eurofusion facility ITM-gateway at Garching, Germany. The typical power consumption per node on this ma- 


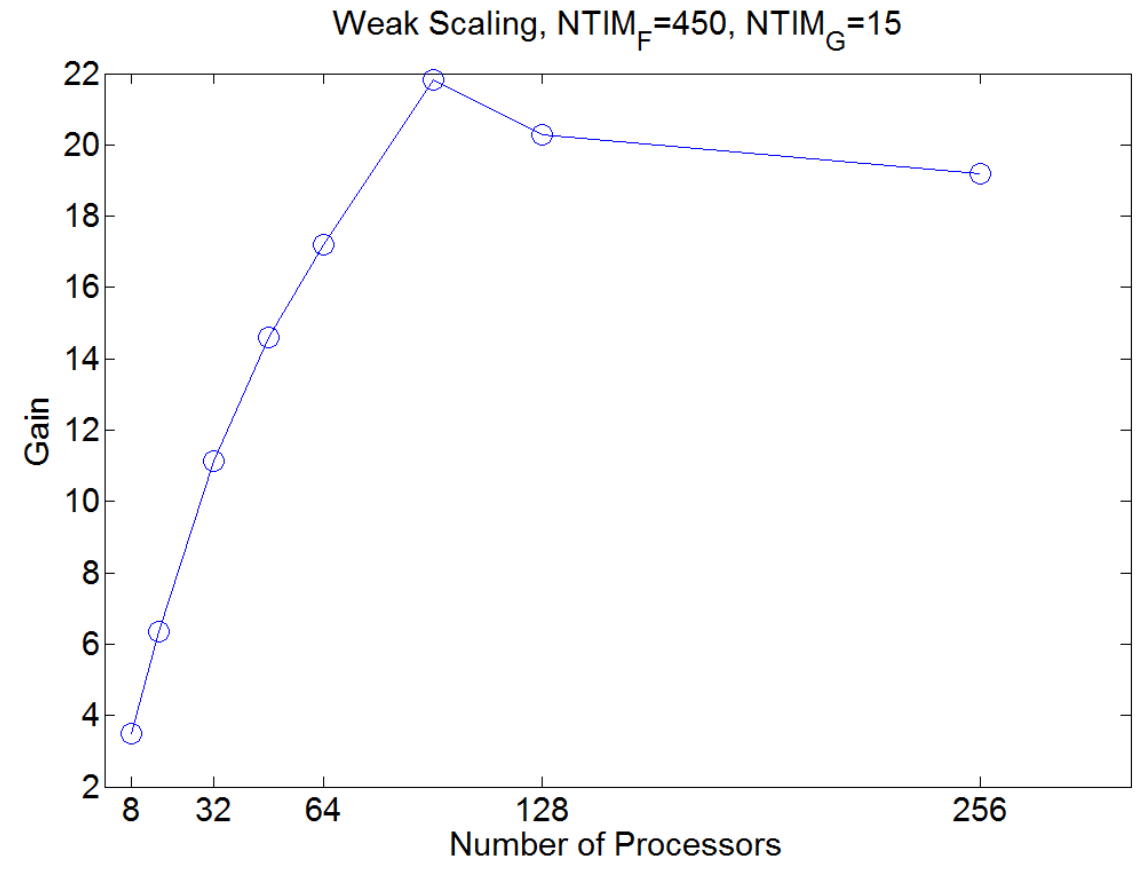

Figure 14: Weak scaling studies. Here $N T I M_{F}=450$ and $N T I M_{G}=15$.

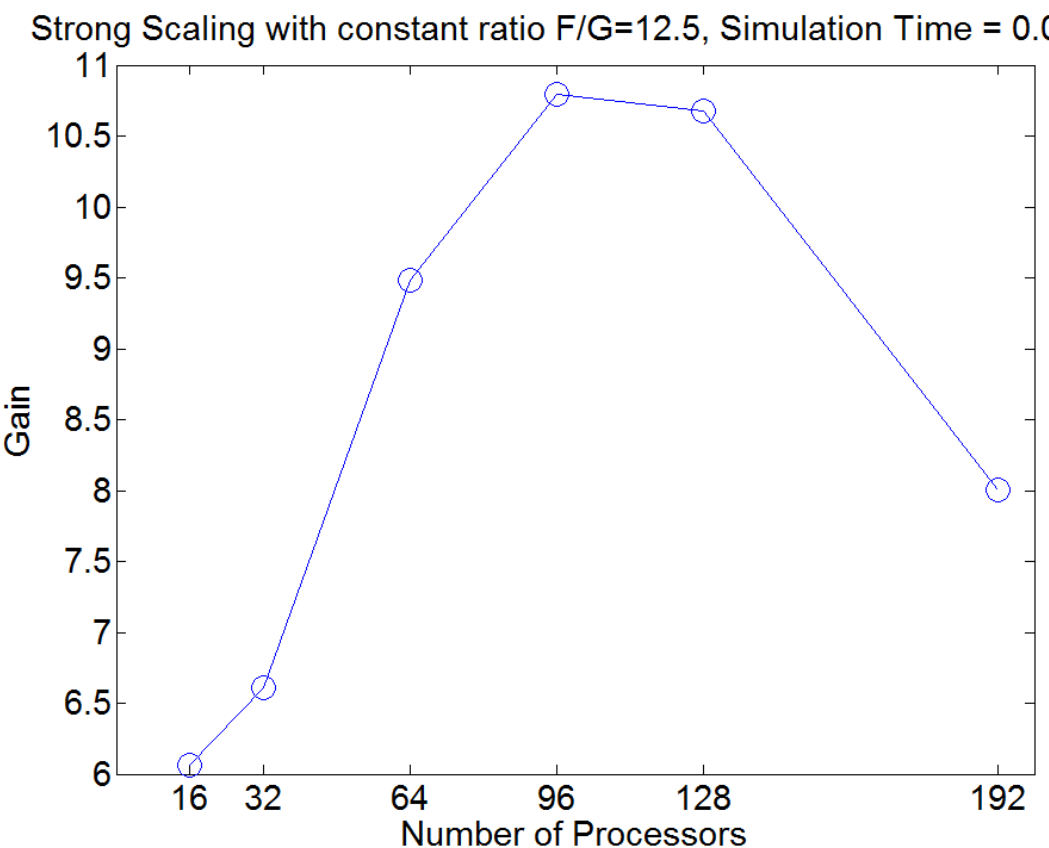

Figure 15: Strong scaling studies. The gain decreases at high processor count when the size of the time slice solved per processor is significantly reduced. 
chine was $186.88 \mathrm{~W}$. With 16 cores per node, the energy consumption per core was $11.68 \mathrm{~W}$. For a case of computational gain of 15.9 with 64 processors, the parareal version uses about 4.03 times more energy than the serial run for the same task. A measure of the energy consumption may also be obtained by computing the inverse of the efficiency of the parallelism. Efficiency is defined as:

$$
\text { Efficiency }=\frac{\text { computational gain }}{\text { processor count }}
$$

So, with a gain of 15.9 for 64 processors, the efficiency is $24.8 \%$.

\subsection{Parareal gain with IPS framework}

The IPS (Integrated Plasma Simulator) framework was used to carry out the parareal implementation in this work. This allowed an event-based approach which positively impacted the computational gain.

For a long time series divided into $\mathrm{N}$ slices using $\mathrm{N}$ processors, let $t_{G}$ be the time taken to perform the coarse $(\mathrm{G})$ computation on a single time slice and let $t_{F}$ be the same for a fine computation on a single time slice as well. If at iteration $\mathrm{k}, n_{c}$ slices have converged then $n_{k}=N-n_{c}$ slices remain to perform a $\mathrm{G}$ and $\mathrm{F}$ calculation. In a traditional, sequential MPI implementation, with $\mathrm{G}$ being a serial process and $\mathrm{F}$ being performed in parallel, the wallclock time required per iteration $\mathrm{k}$ can then be stated as:

$$
t_{k}=t_{G} * n_{k}+t_{F}
$$

If $\mathrm{K}$ is the total number of iterations required for convergence of all $\mathrm{N}$ time slices, then summing across all iterations gives the total time for a traditional parareal implementation using MPI.

$$
T_{\text {trad }}=\sum_{k=1}^{K} t_{k}
$$

The computational gain for sequential implementation of the parareal algorithm using MPI may then be stated as: 


$$
\text { gain }_{\text {trad }}=\frac{T_{F}}{T_{\text {trad }}}=\frac{t_{F} * N}{T_{\text {trad }}}
$$

The fact that the event-based approach significantly improves performance is illustrated in fig 16 where the plot for the event-based implementation using IPS (in red) is generated by actual simulation results discussed in section 3.1 The other plot (in black) is a theoretical estimate using eq. (10) of the sequential MPI implementation of the algorithm. It may be added that the theoretical calculation of computational gain in fig 16 ignores MPI communication overheads. This clearly demonstrates the efficiency of the framework, where the computational gain grows almost linearly, while with traditional MPI, the gain would have flattened at a much smaller value.

As already mentioned in section 2.3 the implementation of the parareal scheme to any case is simplified by the use of the framework. There are no massive changes to the original physics code (SOLPS) that are required, and the user can focus on varying and optimizing the coarse solvers instead of the numerics of the scheme.

\section{Conclusion}

This work has shown that time parallelization greatly contributes to speeding up of edge plasma simulations. The parareal algorithm has been applied to the SOLPS-Eirene code package and a gain larger than 20 has been achieved which is significant for edge simulation in plasmas. It has also been shown that the use of event-based parareal via the IPS framework has a very strong impact on the computational gain. The parareal approach was explored on simulations of two different machines performing experiments of magnetic fusion - namely the DIII-D and MAST tokamaks. Both studies generated promising results. The parareal algorithm, which uses a predictor-corrector technique, requires a coarse and a fine solver. The fine solver uses SOLPS B2.5 and Eirene code packages and is relatively computationally intensive. The choice of the coarse solver as in any work involving the parareal algorithm, is non trivial. Two 


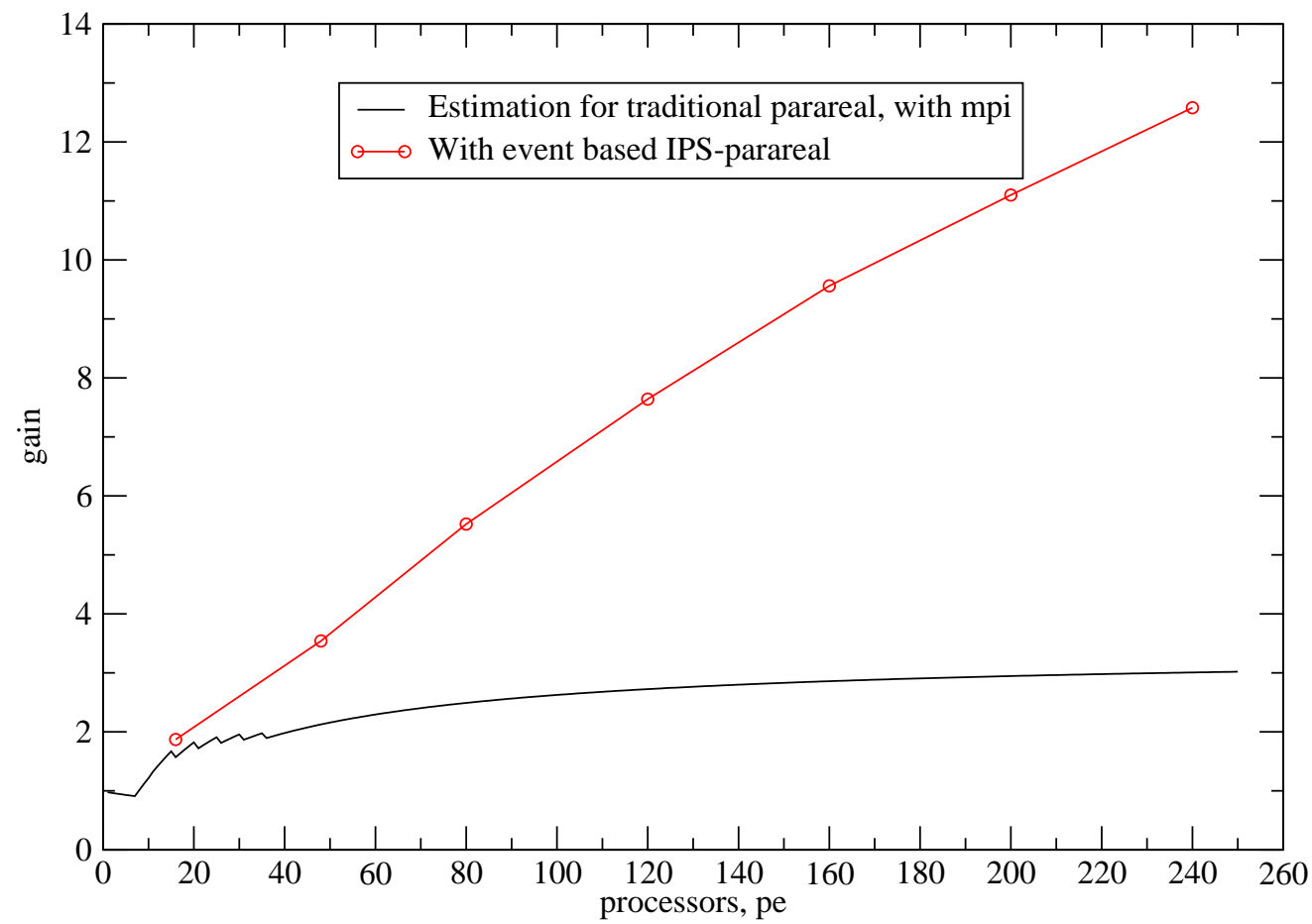

Figure 16: The computational gain is significantly larger when the event-based parareal is applied using the IPS framework. A theoretical estimate of the gain using traditional, sequential MPI implementation shows a much lower value and quick saturation with processor numbers. 
coarse solvers have been explored in this work. The Eirene package, which solves the Boltzman equation for a kinetic treatment of neutral particles, has been replaced by a simpler fluid neutral model to serve as a coarse predictor. This has allowed significant speed-up of the code, but a strong sensitivity to the size of the time slice (number of simulation time steps solved per processor) has been observed. A size of 10 appears to be the optimum value for the cases considered. The second coarse solver uses a reduced grid model accompanied by bigger simulation time steps $(\mathrm{dt})$. This coarse solver appears to have a more robust performance than the one discussed earlier and it, too, leads to significant computational gain. Scaling studies have also been performed. It was observed that the computational gain using the parareal algorithm depends on the size of time slices solved on each processor. The computational gains obtained may also be further improved by combining the two coarse solvers along with additional levels of parallelism, such as, space parallelization. The idea of the coarse solvers explored in this work may be expanded to other areas of computational physics. A reduced mesh for the coarse domain may be explored wherever applicable although limitations to their extent may be expected. Using reduced physics for the coarse model should be attempted with care, as has been done here, to ensure that the parareal correction at every iteration prevents the solution from deviating from the fine simulation.

\section{Acknowledgements}

This work has been funded within the framework of the EUROfusion Consortium and has received funding from the Euratom research and training programme 2014-2018 under grant agreement No 633053. The views and opinions expressed herein do not necessarily reflect those of the European Commission. This work has been part-funded by the RCUK Energy Programme [grant number EP/I501045]. To obtain further information on the data and models underlying this paper please contact PublicationsManager@ccfe.ac.uk. Also, the views and opinions expressed herein do not necessarily reflect those of the ITER 
Organization. The authors are also extremely thankful to the consultants at the EUROfusion Gateway cluster hosted by IPP Garching, Germany. The authors would also like to thank John Canik for providing the test case for the DIII-D simulations and Tim Hender for the valuable discussions and inputs.

\section{References}

[1] J. Lions, Y. Maday, G. Turinici, A "parareal" in time discretization of PDE's, Comptes Rendus de l'Académie des Sciences - Series I - Mathematics 332 (7) (2001) 661-668.

[2] L. Baffico, S. Bernard, Y. Maday, G. Turinici, G. Zérah, Parallel in time molecular dynamics simulations, Phys. Rev. E 66 (5) (2002) 057706.

[3] G. Bal, Y. Maday, A parareal time discretization for non-linear PDEs with application to the pricing of an American put, Vol. 23 of Lect. Notes Comput. Sci. Eng., Springer, Berlin, 2002, p. 189202.

[4] G. Bal, Parallelization in time of (stochastic) ordinary differential equations (2003).

URL http://www . columbia.edu/ gb2030/PAPERS/paralleltime.pdf

[5] P. F. Fischer, F. Hecht, Y. Maday, A parareal in time semi-implicit approximation of the Navier-Stokes equations, in: Proceedings of Fifteen International Conference on Domain Decomposition Methods, Vol. 40, Springer Verlag, 2004, pp. 433-440.

[6] M. J. Gander, E. Hairer, Nonlinear convergence analysis for the parareal algorithm, in: Domain Decomposition Methods in Science and Engineering XVII, Vol. 60, Springer, 2008, p. 45.

[7] I. Garrido, M. S. Espedal, G. E. Fladmark, A convergent algorithm for time parallelization applied to reservoir simulation, in: Proceedings of Fifteen International Conference on Domain Decomposition Methods, Vol. 40, Springer, Berlin, 2004, p. 469. 
[8] G. Staff, G. Rønquist, Stability of the parareal algorithm, in: Proceedings of Fifteen International Conference on Domain Decomposition Methods, Springer Verlag, 2003, pp. 449-456.

[9] D. Samaddar, D. E. Newman, R. Sánchez, Parallelization in time of numerical simulations of fully-developed plasma turbulence using the parareal algorithm, Journal of Computational Physics 228 (2010) 6558.

[10] J. Reynolds-Barredo, D. E. Newman, R. Sánchez, D. Samaddar, L. A. Berry, W. R. Elwasif, Mechanisms for the convergence of time-parallelized, parareal turbulent plasma simulations, Journal of Computational Physics $231(2012) 7851$.

[11] D. Samaddar, T. Casper, S. Kim, L. A. Berry, W. R. Elwasif, D. B. Batchelor, W. Houlberg, Application of the parareal algorithm to advanced operation scenario simulations of ITER plasma using the CORSICA code, Applied Mathematics and Computation (submitted).

[12] A.-M. Baudron, J.-J. Lautard, Y. Maday, M. K. Riahi, J. Salomon, Parareal in time 3D numerical solver for the LWR Benchmark neutron diffusion transient model, Journal of Computational Physics (submitted).

[13] P. C. Stangeby, The plasma boundary of magnetic fusion devices, Vol. 7, Taylor \& Francis, 2000.

[14] J. Wesson, Vol. 149, Oxford University Press, 2011.

[15] X. Q. Xu, R. H. Cohen, Scrape-Off Layer Turbulence Theory and Simulations, Contrib.Plasma Phys. 38 (1-2) (1998) 158.

[16] X. Q. Xu, R. H. Cohen, T. D. Rognlien, J. R. Myra1, A fully implicit, time dependent 2-D fluid code for modeling tokamak edge plasmas, Journal of Nuclear Materials 7 (2000) 1951.

[17] T. Rognlien, J. Milovich, M. Rensink, G. Porter, Scrape-Off Layer Turbulence Theory and Simulations, Contrib.Plasma Phys. 196-198 (1992) 347. 
[18] P. Stangeby, J. Elder, Calculation of observable quantities using a divertor impurity interpretive code, DIVIMP, Journal of Nuclear Materials 196 (1992) 258.

[19] A. Kirschner, V. Philipps, J. Winter, U. Kögler, Simulation of the plasmawall interaction in a tokamak with the Monte Carlo code ERO-TEXTOR, Nucl. Fusion 40 (5) (2000) 989.

[20] J. Heikkinen, T. Kiviniemi, T. Kurki-Suonio, A. Peeters, S. Sipilä, Particle Simulation of the Neoclassical Plasmas, Journal of Comp. Phys. 173 (2) (2001) 527.

[21] O. E. Garcia, N. H. Bian, Bursting and large-scale intermittency in turbulent convection with differential rotation, Phys. Rev. E 68 (2003) 047301.

[22] R. Schneider, X. Bonnin, K. Borrass, D. P. Coster, H. Kastelewicz, D. Reiter, V. A. Rozhansky, B. J. Braams, Plasma Edge Physics with B2-Eirene, Contrib.Plasma Phys. 46 (1-2) (2006) 3.

[23] P. B. D. Reiter, M. Baelmans, The EIRENE and B2-EIRENE Codes, Fusion Science and Technology 47 (2) (2005) 172.

[24] Y. Feng, F. Sardei, J. Kisslinger, P. Grigull, K. McCormick, D. Reiter, 3D Edge Modeling and Island Divertor Physics, Contrib. Plasma Phys. 44 (1) (2004) 57.

[25] R. Simonini, G. Corrigan, G. Radford, J. Spence, A. Taroni, Models and Numerics in the Multi-Fluid 2-D Edge Plasma Code EDGE2D/U, Contrib.Plasma Phys. 34 (2-3) (1994) 368.

[26] H. Bufferand, G. Ciraolo, L. Isoardi, G. Chiavassa, F. Schwander, E. Serre, N. Fedorczak, P. Ghendrih, P. Tamain, Applications of SOLEDGE-2D code to complex SOL configurations and analysis of Mach probe measurements, Journal of Nuclear Materials 415 (1, Supplement) (2011) S589S592. 
[27] A. S. Kukushkin, H. D. Pacher, Divertor modelling and extrapolation to reactor conditions, Plasma Phys. Control. Fusion 44 (6) (2002) 931.

[28] A. Loarte, et al., Power and particle control, Nucl. Fusion 47 (2007) S203.

[29] A. S. Kukushkin, H. D. Pacher, V. Kotov, G. Pacher, D. Reiter, Finalizing the ITER divertor design: The key role of SOLPS modeling, Fusion Engineering and Design 86 (12) (2011) 2865.

[30] G. Herre, P. Grigull, R. Schneider, B2-Eirene code modelling of an island divertor, Journal of Nuclear Materials 266-269 (1999) 1015-1019.

[31] X. Bonnin, R. Schneider, D. Coster, V. Rozhansky, S. Voskoboynikov, Electric fields and currents in an island divertor configuration, Journal of $\mathrm{Nu}-$ clear Materials 829835, 14th Int. Conf. on Plasma-Surface Interactions in Controlled Fusion Devices (2001) 7 .

[32] R. A. Pitts, A. Kukushkin, A. Loarte, A. Martin, M. Merola, C. E. Kessel, V. Komarov, M. Shimada, Status and physics basis of the ITER divertor, Physica Scripta 2009 (2009) T138.

[33] W. Elwasif, D. Bernholdt, A. Shet, S. Foley, R. Bramley, D. B. Batchelor, L. A. Berry, The Design and Implementation of the SWIM Integrated Plasma Simulator, in: Distributed and Network-based Processing (PDP), Pisa, Italy, 2010.

[34] W. R. Elwasif, S. Foley, D. Bernholdt, L. A. Berry, D. Samaddar, D. E. Newman, R. Sanchez, A dependency-driven formulation of parareal: parallel-in-time solution of PDEs as a many-task application, in: MTAGS '11 Proceedings of the 2011 ACM international workshop on Many task computing on grids and supercomputers, ACM New York, NY, USA, 2011, p. 15.

[35] L. A. Berry, W. R. Elwasif, J. Reynolds-Barredo, D. Samaddar, R. Sanchez, D. E. Newman, Event-based parareal: A data-flow based implementation of parareal, Journal of Comp. Phys. 231 (17) (2012) 5945. 
[36] C. Farhat, M. Chandesris, Time-decomposed parallel time-integrators: theory and feasibility studies for fluid, structure, and fluid-structure applications., Int. J. Numer. Meth. Engng. 58 (9) (2003) 1397.

[37] M. Emmett, M. L. Minion, Toward an efficient parallel in time method for partial differential equations, Communications in Applied Mathematics and Computational Science 7 (1) (2012) 105.

[38] R. Speck, D. Ruprecht, M. Emmett, M. Bolten, R. Krause, "A space-time parallel solver for the three-dimensional heat equation., in: Advances in Parallel Computing, Volume 25: Parallel Computing: Accelerating Computational Science and Engineering (CSE), 2014.

[39] A. J. Christlieb, R. D. Haynes, B. W. Ong, A parallel space-time algorithm, SIAM Journal on Scientific Computing. 34 (5) (2012) C233-C248.

[40] S. I. Braginskii, Transport Processes in a Plasma, Vol. 1, Consultants Bureau, New York, 1965, p. 205.

[41] E. Havlícková, W. Fundamenski, F. Subba, D. Coster, M. Wischmeier, G. Fishpool, Benchmarking of a 1D scrape-off layer code SOLF1D with SOLPS and its use in modelling long-legged divertors, Plasma Phys. Control. Fusion 55 (6) (2013) 065004.

[42] V. Rozhansky, S. Voskoboynikov, E. Kaveeva, D. Coster, R. Schneider, Simulation of tokamak edge plasma including self-consistent electric fields, Nuclear Fusion 41 (4) (2001) 387.

[43] D. P. Coster, Reduced physics models in SOLPS for reactor scoping studies, Contributions to Plasma Physics 56 (2016) 790-795.

[44] D. Coster, X. Bonnin, B. Braams, D. Reiter, R. Schneider, the ASDEX Upgrade Team, Simulation of the Edge Plasma in Tokamaks, Physica Scripta 2004 (T108) (2004) 7. 
[45] D. P. Coster, Detachment physics in SOLPS simulations, Journal of Nuclear Materials - Proceedings of the 19th International Conference on PlasmaSurface Interactions in Controlled Fusion. Volume 415, Issue 1, Supplement (2011) S545S548.

[46] X. Bonnin, H. Bürbaumer, R. Schneider, D. Coster, F. Aumayr, H. P. Winter, The Two-Mesh Grid Refinement Method for the B2 Code, Contributions to Plasma Physics 42 (2) (2002) 175.

[47] K. Ghoos, W. Dekeyser, G. Samaey, P. Börner, D. Reiter, M. Baelmans, Accuracy and Convergence of Coupled Finite-Volume / Monte-Carlo Codes for Plasma Edge Simulations, Contributions to Plasma Physics 56 (6-8) (2016) 616-621.

[48] M. L. Minion, A Hybrid Parareal Spectral Deferred Corrections Method,, Communications in Applied Mathematics and Computational Science 5 (2010) 265-301.

[49] E. Aubanel, Scheduling of Tasks in the Parareal Algorithm, Parallel Computing 37 (3) (2011) 172-182. 